\title{
Special Issue: Methods in Computational Biology
}

\author{
Ross P. Carlson ${ }^{1, *}$ and Herbert M. Sauro ${ }^{2, *}$ \\ 1 Department of Chemical and Biological Engineering, Montana State University, Bozeman, MT 59717, USA \\ 2 Department of Bioengineering, University of Washington, Seattle, WA 98195-5061, USA \\ * Correspondence: rossc@montana.edu (R.P.C.); hsauro@uw.edu (H.M.S.); Tel.: +406-994-3631 (R.P.C.); \\ +206-685-2119 (H.M.S.)
}

Received: 8 April 2019; Accepted: 8 April 2019; Published: 11 April 2019

check for updates

Biological systems are multiscale with respect to time and space, exist at the interface of biological and physical constraints, and their interactions with the environment are often nonlinear. These systems are being quantified in ever increasing detail using rapidly developing omics technologies; yet, it is difficult to predict the dynamic and spatial behavior of even the simplest model systems. Computational biology approaches are essential for leveraging the omics data to develop and test new theories on biological organization. This is a major challenge for the life sciences, including the medical, environmental, and bioprocess fields.

A primary goal of this Special Issue "Methods in Computational Biology" is the communication of computational biology methods, which can extract biological design principles from complex data, described in enough detail to permit reproduction of the results. This issue integrates highly interdisciplinary researchers such as biologists, computer scientists, engineers and mathematicians to advance biological systems analysis. A summary of the contributions to the Special Issue are provided in the following section; many of the contributions are mentioned more than once because their content includes themes that fall under multiple categories.

\section{Reviews of Computational Methods}

The Special Issue includes two contributions which review and synthesize important aspects of computational analysis. In Hunt et al. [1], the authors summarize, organize and provide examples of seven different 'mechanism-oriented' model types and discuss how they can be employed to analyze biological phenomena. Coverage includes not only a mathematical description, but also solvers and simulation considerations. Norton et al. [2] provide a thorough review of agent-based modeling of tumor cells, tumor cell heterogeneity as well as tumor interactions with host immune system components and local physical environments.

\section{Computational Analysis of Biological Dynamics: From Molecular to Cellular to Tissue/Consortia Level}

Life is an inherently dynamic process. The Special Issue includes analysis of dynamic processes on molecular, cellular, tissue and microbial consortia size scales. A comparison of the different size scales identifies mathematical and computational approaches that span scales. Porubsky and Sauro [3] examine molecular level processes_-for instance, gene networks—and present methodologies for optimizing parameters necessary to obtain models that exhibit oscillating behavior. Erhardt [4] examines cellular scale systems and the role of calcium-induced oscillations in cardiac cells and its role in cardiac arrhythmis. The study applies a number of theories and methods including bifurcation theory, numerical bifurcation analysis, and geometric singular perturbation theory to study nonlinear multi time scale systems. Pool et al. [5] studies intra- and extracellular processes associated with cholesterol and lipoprotein metabolism and how intervention strategies such as statins or diet can influence metabolism. Farzan and Ierapetritou [6] report on multicellular scale systems and analyze interactions between mammalian cells and the bioreactor environment with the ultimate goal of 
optimizing bioprocess applications. Norton et al. [2] study systems on the cellular and tissue scales and examine the interactions between tumor cells, host immune cells and local microenvironments. Phalak and Henson [7] study a multicellular scale system quantifying the dynamic interactions between multiple microorganisms, including the exchange of metabolites, and the role of time and space on microbial infections.

\section{The Interface of Biotic and Abiotic Processes}

Life occurs at the interface of biological and physical constraints. Biological processes, including metabolism, are constrained by physical processes such as chemical transport to and from the cell. Phalak and Henson [7] analyze how assemblages of different microorganisms can organize along chemical gradients established by an imbalance between biological reaction rates and abiotic diffusion rates. These gradients lead to spatial distributions of cell types and often enhanced system robustness. Farzan and Ierapetritou [6] consider the interface of mammalian cells and convective transport processes which ultimately influence the local chemical, thermal and mechanical environments. The work also discusses computational optimization and selection of solvers for these types of modeling applications.

\section{Processing of Large Data Sets for Enhanced Analysis}

Modern biology is rapidly becoming a study of large sets of data. Roberts et al [8] analyze tools for extracting additional information from microRNA extracted from breast cancer by measuring 50,000 recurrent editing sites. The data identifies the presence of additional levels of complexity in microRNAs which influences how the molecules interact with target mRNA.

Representing the output from computational biology efficiently, in a manner that facilitates communication, is often difficult. Rose and Mazat [9] present software that enables the visualization of metabolic flux data using a graphical user interface that permits rapid and simplified formatting of data.

\section{Parameters Optimization and Measurements}

Computational representations of life require parameters. Parameter identification is a major challenge and a focus of many studies. The Special Issue includes contributions which focus on optimizing parameters required to represent biphasic systems, including generalized mass action networks, relevant to gene signaling and metabolite networks [3], as well as calcium-induced oscillation in cardiac cells [4]. Beck et al. [10] provide detailed methods for experimentally measuring key parameters required for genome-scale metabolic models, including the biomass synthesis reaction. The authors then demonstrate how different biomass parameters produce very different results based on the interaction of electron balances and metabolism.

This Special Issue is coordinated with the Metabolic Pathway Analysis 2017 conference held in Bozeman, MT and Interagency Modeling and Analysis Group (IMAG) MultiScale Modeling (MSM) working groups (https:/ / www.imagwiki.nibib.nih.gov/).

\section{References}

1. Hunt, C.; Erdemir, A.; Lytton, W.; Mac Gabhann, F.; Sander, E.; Transtrum, M.; Mulugeta, L. The Spectrum of Mechanism-Oriented Models and Methods for Explanations of Biological Phenomena. Processes 2018, 6, 56. [CrossRef]

2. Norton, K.A.; Gong, C.; Jamalian, S.; Popel, A.S. Multiscale Agent-Based and Hybrid Modeling of the Tumor Immune Microenvironment. Processes 2019, 7, 37. [CrossRef] [PubMed]

3. Porubsky, V.L.; Sauro, H.M. Application of Parameter Optimization to Search for Oscillatory Mass-Action Networks Using Python. Processes 2019, 7, 163. [CrossRef] 
4. Erhardt, A. Early After depolarisations Induced by an Enhancement in the Calcium Current. Processes 2019, 7, 20. [CrossRef]

5. Pool, F.; Sweby, P.; Tindall, M. An Integrated Mathematical Model of Cellular Cholesterol Biosynthesis and Lipoprotein Metabolism. Processes 2018, 6, 134. [CrossRef]

6. Farzan, P.; Ierapetritou, M. A Framework for the Development of Integrated and Computationally Feasible Models of Large-Scale Mammalian Cell Bioreactors. Processes 2018, 6, 82. [CrossRef]

7. Phalak, P.; Henson, M.A. Metabolic Modeling of Clostridium difficile Associated Dysbiosis of the Gut Microbiota. Processes 2019, 7, 97. [CrossRef]

8. Roberts, J.; Patterson, D.; King, V.; Amin, S.; Polska, C.; Houserova, D.; Crucello, A.; Barnhill, E.; Miller, M.; Sherman, T.; et al. ADAR Mediated RNA Editing Modulates MicroRNA Targeting in Human Breast Cancer. Processes 2018, 6, 42. [CrossRef] [PubMed]

9. Rose, T.; Mazat, J.P. FluxVisualizer, a Software to Visualize Fluxes through Metabolic Networks. Processes 2018, 6, 39. [CrossRef]

10. Beck, A.; Hunt, K.; Carlson, R. Measuring Cellular Biomass Composition for Computational Biology Applications. Processes 2018, 6, 38. [CrossRef]

(C) 2019 by the authors. Licensee MDPI, Basel, Switzerland. This article is an open access article distributed under the terms and conditions of the Creative Commons Attribution (CC BY) license (http://creativecommons.org/licenses/by/4.0/). 